\title{
Plane-by-Plane Inscription of Grating Structures in Optical Fibers
}

\author{
Ping Lu ${ }^{(\mathbb{D}}$, Stephen J. Mihailov ${ }^{(}$, Senior Member, IEEE, Fellow, OSA, Huimin Ding, Dan Grobnic, \\ Robert B. Walker, David Coulas, Cyril Hnatovsky, and Andrei Yu Naumov
}

\begin{abstract}
Plane-by-plane fabrication of fiber Bragg gratings in optical fibers using a short-pulse femtosecond IR laser is proposed and demonstrated. By incorporating a cylindrical lens in the fabrication setup, a plane of index modification can be directly inscribed in the fiber core by a single laser pulse. The planes of index modification are produced in the core of a free-standing fiber without an oil immersion objective. This plane-by-plane method simplifies the direct grating inscription process and allows for the fabrication of complicated grating structures.
\end{abstract}

Index Terms - Cylindrical lens, direct inscription, femtosecond laser, fiber Bragg gratings, plane-by-plane.

\section{INTRODUCTION}

$\mathbf{F}$ EMTOSECOND lasers have been used to fabricate Bragg grating structures in various fiber materials due to the lasermaterial interaction via nonlinear photoabsorption and photoionization mechanisms. Phase mask scanning [1], [2] and direct inscription point-by-point $(\mathrm{PbP})$ method [3]-[9] are the two common techniques that have been widely used for grating inscription using ultrafast infrared lasers. In the phase mask approach, the grating parameters, such as Bragg wavelength, grating length, chirp, phase shift etc., are determined by the specific phase mask, which can be costly to produce when long and complex grating structures are required. By directly focusing the femtosecond laser beam into the fiber core, direct inscription methods have been demonstrated to be more flexible for writing grating structures with different Bragg resonant wavelengths, chirp and cladding mode coupling etc. However, the modified index regions produced by direct inscription methods are microvoids which are usually much smaller than the fiber core diameter resulting weak mode coupling coefficient [10]. For a fiber Bragg grating (FBG) inscribed in an optical fiber, the transverse coupling coefficient, $K_{j k}^{t}(z)$, between modes $j$ and $k$

Manuscript received July 24, 2017; revised August 30, 2017; accepted September 3, 2017. Date of publication September 7, 2017; date of current version February 24, 2018. (Corresponding author: Ping Lu.)

The authors are with the National Research Council Canada, Ottawa, ON K1A 0R6, Canada (e-mail: Ping.Lu@nrc-cnrc.gc.ca; Stephen.Mihailov@nrc-cnrc. gc.ca; Huimin.Ding@nrc-cnrc.gc.ca; Dan.Grobnic@nrc-cnrc.gc.ca; Robert. Walker2@nrc-cnrc.gc.ca; David.Coulas@nrc-cnrc.gc.ca; Cyril.Hnatovsky@ nrc-cnrc.gc.ca; Andrei.Naumov@nrc-cnrc.gc.ca).

Color versions of one or more of the figures in this paper are available online at http://ieeexplore.ieee.org.

Digital Object Identifier 10.1109/JLT.2017.2750490 is [11]

$$
\begin{aligned}
K_{j k}^{t}(z)= & \frac{\pi}{\lambda} \iint_{\infty} n(x, y, z) \Delta n(x, y, z) \\
& \cdot \vec{e}_{k t}(x, y) \cdot \vec{e}_{j t}^{*}(x, y) d x d y
\end{aligned}
$$

where $n(x, y, z)$ is the refractive index of the fiber, $\Delta n(x, y, z)$ is the induced index change by laser radiation, $\vec{e}_{k t}(x, y)$ and $\vec{e}_{j t}(x, y)$ are the transverse fields of modes $k$ and $j$ respectively. From (1) we can see that the mode coupling coefficient is proportional to the overlap integral of the index change $\Delta n(x, y, z)$ and the transverse mode field. In the case that a fiber Bragg grating is inscribed in a single mode fiber with a phase mask, the induced index change $\Delta n$ is usually in the form of sinusoidal function and is uniform across the fiber core, then the "ac" component, $\kappa$, of coupling coefficient at wavelength, $\lambda$, can be simplified as [11]

$$
\kappa=\frac{\pi}{\lambda} \nu \overline{\Delta n}_{e f f} \stackrel{\sim}{=} \frac{\pi}{\lambda} \nu \Gamma \overline{\Delta n}_{c o}
$$

where $\nu$ is the fringe visibility of index change, $\overline{\Delta n}_{e f f}$ is the is the "dc" index change spatially averaged over a grating period, $\Gamma$ is the mode power confinement factor in the fiber core, and $\overline{\Delta n}_{c o}$ is the average index change in the fiber core.

In the point-by-point direct FBG inscription method, the coupling coefficient $\kappa$ cannot be simplified as described in (2) because the index change is usually not sinusoidal along the fiber and not uniform across the fiber core. The dimensions of the region of the index modification $\Delta n$ in the fiber core is much smaller $(\sim \mu \mathrm{m})$ compared to that produced by the phase mask approach. There is only a small overlap between the mode field propagating along the fiber core and the dimension of the index change resulting in weaker mode coupling according to (1). The small overlap causes a weak grating reflectivity for a given index change. From (1) we can also see that if the region of the induced index change $\Delta n$ is not located at the center of the single mode fiber core, the coupling coefficient will drop because the peak intensity of mode field is at the center of the core in single mode fibers. As a result, the alignment requirement for the point-by-point method is more critical than that for the phase mask technique.

For a given type of fiber, the only way to increase the coupling coefficient is to increase the amplitude of the index change $\Delta n$ and/or the dimension of the region of the index change. Femtosecond lasers can produces very high index change and strong type II gratings in various fibers [12], but high scattering losses 
are introduced as well. Several approaches have been reported to increase the coupling coefficient in order to produce strong grating reflectivities while producing low scattering loss. Zhou et al. [5] showed a line-by-line method to increase the index change dimension by moving the oil-immersion objective perpendicularly to the fiber axis so that a line instead of a point of index change is produced across the fiber core. After one line is made, the fiber is moved along the fiber axis at a distance of the grating period and then another line of index change is made. Strong higher order gratings were fabricated by using this technique but with very high scattering loss $(1.2 \mathrm{~dB} / \mathrm{cm})$, which is not desirable for chirped gratings. Williams et al. [6] presented a continuous core-scanning technique where a piezo stage was driven by a sine wave, resulting in a sinusoidal index change across the core. Strong first order gratings with low induced scattering loss were fabricated by using technique. The fabrication process however is complicated and time consuming when long gratings are inscribed. Strong cladding modes were also observed. In the two methods described above, oil-immersion objectives were used and the fiber is also immersed in the oil during grating fabrication. The process is further complicated by requiring the fiber to be cleaned after the grating inscription which weakens the mechanical strength of the grating and will make it harder to recoat the fiber. Without using an oilimmersion based setup, Lai et al. [9] presented the technique of beam profiling using a slit by which grating pitches were inscribed in free-standing fiber through a pitch-by-pitch process. Strong gratings were fabricated but the induced scattering loss is still high $(0.2 \mathrm{~dB} / \mathrm{cm})$ that is not acceptable for some applications, such as long and large chirped gratings.

In this work, we report a modified approach to the point-bypoint technique where by adding a cylindrical lens to the exposure setup before the final microscope objective, the cylindrical shape of the focal volume normally produced by the microscope objective alone is instead transformed into a planar strip or plane [13]-[15]. We will show that the proposed approach produces a grating structure in the fiber core that is written plane-by-plane with single laser pulses. This approach effectively increases the mode coupling in the fiber core simplifying the fabrication of strong Type I and Type II direct write gratings. The grating planes are inscribed in the core of free-standing fiber without using oil-immersion objective based approach, which simplifies the grating fabrication setup and makes possible the fabrication of long grating structures such as chirped and tilted gratings. Such structures are important for fiber laser and sensor applications involving fiber gratings.

\section{Setup of FABRicAting GratingS By Plane-BY-Plane Method}

The diagram in Fig. 1 shows the setup for grating fabrication in an optical fiber through plane-by-plane method. It is similar to the setup for point-by-point grating inscription except that a long focal length cylindrical lens was added to the beam path with its focal point positioned at the same location as that of the microscope objective [13]. The regeneratively amplified Ti:sapphire laser (Spitfire, Coherent) operates at the wavelength

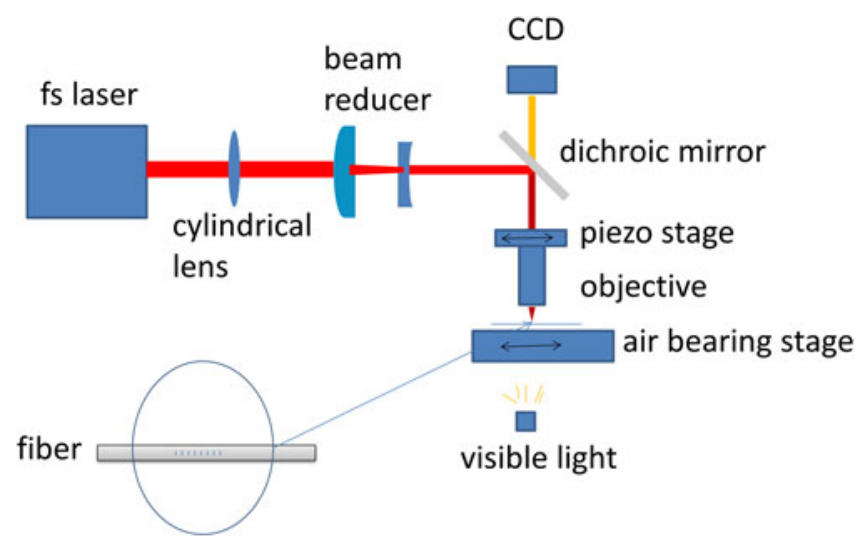

Fig. 1. Diagram of the setup for plane-by-plane grating fabrication in optical fibers.

of $800 \mathrm{~nm}$, a repetition rate up to $1000 \mathrm{~Hz}$ and a pulse duration of $120 \mathrm{fs}$. Pulse energies incident on the fiber were between 1.4 to $1.9 \mu \mathrm{J}$. A beam reduction telescope consisting of one planoconvex lens and one plano-concave lens was placed after the cylindrical lens to reduce the laser beam diameter to match the pupil diameter of the microscope objective (50 X/0.6, Nikon). The fiber (SMF-28) was mounted on an air bearing stage (Aerotech) that moves with a positional accuracy of $\pm 10 \mathrm{~nm}$. The objective was mounted on a piezo stage (NanoMax-TS, Melles Griot). By adjusting the axis tilt of the cylindrical lens, the focal line was oriented perpendicular to the fiber axis. When the focal line propagates across the fiber core, a plane of index modification can be produced by a single pulse. As a result, higher mode coupling efficiency and stronger grating strength can be achieved.

\section{RESULTS AND DISCUSSION}

\section{A. Type I Gratings}

Type I gratings were fabricated with a pulse energy of $1.6 \mu \mathrm{J}$. The laser repetition rate was set at $250 \mathrm{~Hz}$ and the fiber was translated at a speed of $132 \mu \mathrm{m} / \mathrm{second}$ resulting in a fundamental 1 st order grating period of $0.528 \mu \mathrm{m}$ and the Bragg wavelength of $1540 \mathrm{~nm}$ (see Fig. 2). The focal length of the cylindrical lens was $50 \mathrm{~cm}$. It was determined that this combination of cylindrical lens and the objective (50 X/0.6, Nikon), resulted in an IR beam line focus of $\sim 11 \mu \mathrm{m}$ in length, however when focused inside a fiber, the line focus is shortened due to the lensing effect of the fiber. Several $10 \mathrm{~mm}$ long gratings were fabricated with various distances between the objective and fiber with the goal of producing strong gratings and low insertion loss. The spectra of a $10 \mathrm{~mm}$ long strong uniform grating are shown in Fig. 2. The grating strength is $25 \mathrm{~dB}$ and the broadband insertion loss in very low $(<0.02 \mathrm{~dB})$ which corresponds to an index modulation $\Delta n=3.8 \times 10^{-4}$. Higher index change (a stronger grating) is expected if the Ge-doped fibers were loaded with $\mathrm{H}_{2}$ or $\mathrm{D}_{2}$ [16]. The size of the focal line at the fiber core was measured with an optical microscope to be around $8 \mu \mathrm{m}$. After passing through the fiber core, the laser beam was ultimately focused in the cladding 


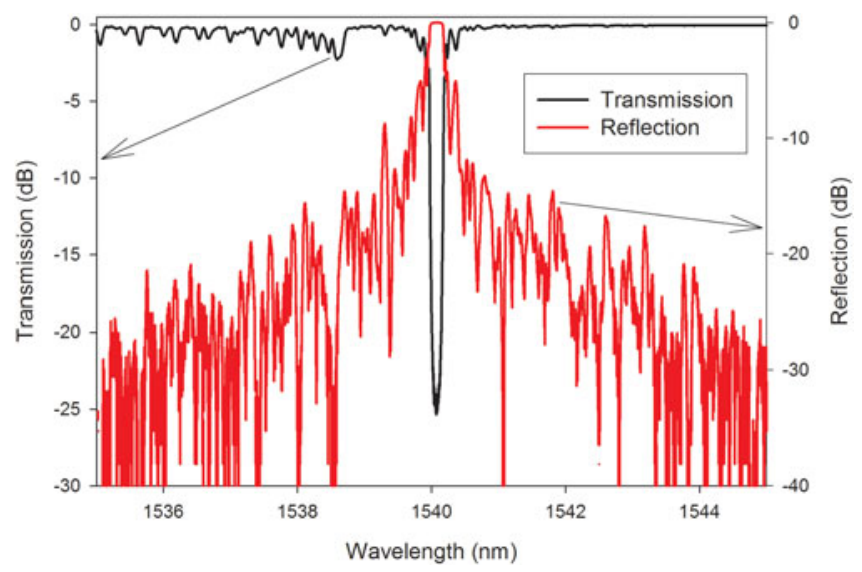

Fig. 2. Spectra of a $10 \mathrm{~mm}$ long type I grating inscribed by plane-by-plane method.

region (a few $\mu \mathrm{m}$ away from the core) creating some damage to the cladding. Compared to the size of index change $(<1 \mu \mathrm{m})$ produced by the objective itself, the advantage of adding the cylindrical lens in the setup to increase mode coupling coefficient is evident, according to (1). The grating was completely erased after baking for an hour at $1000{ }^{\circ} \mathrm{C}$ indicating that type I index change is occurring in the core region. The pulse energy $(1.6 \mu \mathrm{J})$ used to produce the low loss type I grating in this experiment is much higher than the pulse energy used for the point-by-point method [6]. This higher pulse energy is needed because the energy is spread onto a much larger focal volume when the cylindrical lens is used in the setup. As a result, the higher pulse energies produce similar power densities as those produced using the point-by-point approach.

By using this plane-by-plane approach, chirped gratings were also produced. The grating chirp was created by varying slightly the laser repetition rate while the fiber sample was translated at a constant velocity. The RF frequency of $80.216 \mathrm{MHz}$ from the seed laser (Tsunami, Spectra-Physics) was sent to a Synchronization and Delay Generator (SDG Elite, Coherent) which was then divided by an integer called the RF divisor and sent to the pump laser (Empower, Spectra-Physics). The repetition rate can then be tuned by changing the RF divisor, for an example, if the RF divisor is varied from 80216 to 80296 , the laser repetition rate is tuned from $1000 \mathrm{~Hz}$ to $999 \mathrm{~Hz}$. In our experiments, the laser repetition rate was around $250 \mathrm{~Hz}$ by setting the trigger divisor to be 4 . By changing the repetition rate rather than the stage velocity, the perturbation to grating period produced during the changing of air-bear stage velocity can be reduced over long distances up to $260 \mathrm{~mm}$. The spectra of a $20 \mathrm{~mm}$ grating with a chirp rate of $1 \mathrm{~nm} / \mathrm{cm}$ are shown in Fig. 3, the laser repetition rate is from $250 \mathrm{~Hz}$ to $249.6 \mathrm{~Hz}$ in $0.01 \mathrm{~Hz}$ increments while the fiber sample was translated at a constant velocity of $136 \mu \mathrm{m} / \mathrm{s}$.

In order to fabricate strong as well as long gratings with large chirp for sensor applications [17], photo-sensitive fiber or deuterium loaded Ge-doped fiber can be used. Fig. 4 shows the spectrum of a $260 \mathrm{~mm}$ long grating with chirp of $2 \mathrm{~nm} / \mathrm{cm}$ fabricated in deuterium loaded SMF-28 fiber. The fs laser pulse

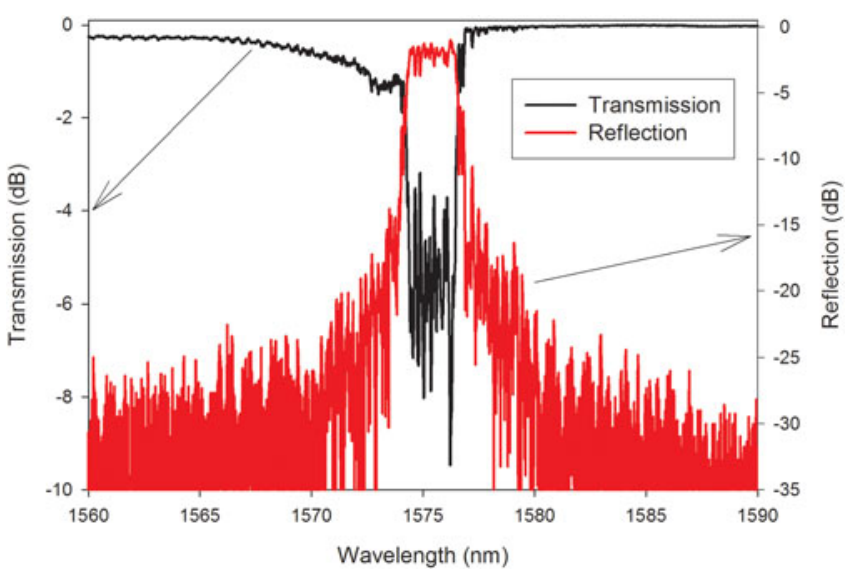

Fig. 3. Spectra of a $20 \mathrm{~mm}$ long type I chirped grating in SMF-28 fiber with a chirp rate of $1 \mathrm{~nm} / \mathrm{cm}$.

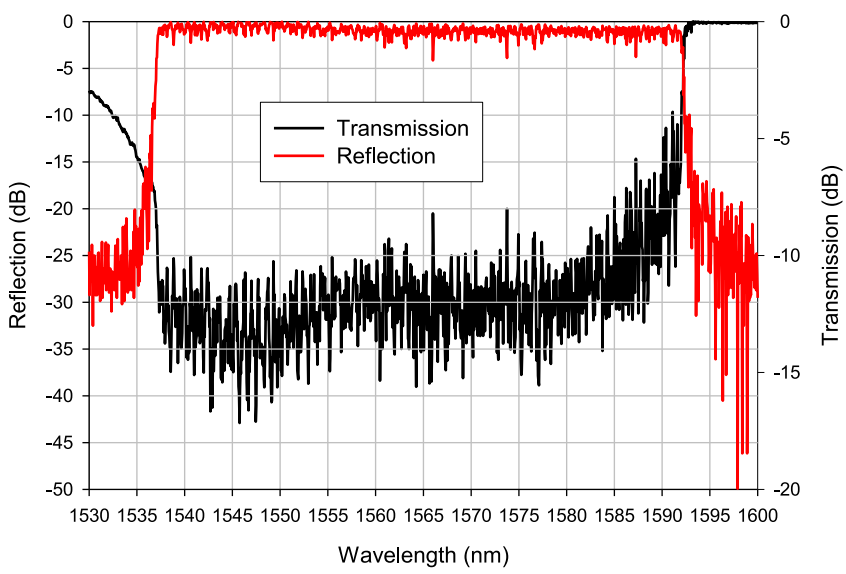

Fig. 4. Spectra of a $260 \mathrm{~mm}$ long and chirped grating with chirp rate of $2 \mathrm{~nm} / \mathrm{cm}$. The total insertion loss is $0.5 \mathrm{~dB}$ for the whole $260 \mathrm{~mm}$ long grating.

energy $(1.6 \mu \mathrm{J})$ was chosen to produce index change as high as possible while maintaining the small insertion loss. The laser induced total insertion loss on the whole $260 \mathrm{~mm}$ long grating is only $0.5 \mathrm{~dB}$ (less than $0.02 \mathrm{~dB} / \mathrm{cm}$ ). The fiber was translated at a speed of $132 \mu \mathrm{m} / \mathrm{s}$, the laser repetition rate was tuned from $250 \mathrm{~Hz}$ to $248.7 \mathrm{~Hz}$ with a step of $0.02 \mathrm{~Hz}$. The step chirped grating was composed 428 uniform grating sections, each section is $608 \mu \mathrm{m}$ in length.

The reflection spectra shown in Figs. 3 and 4 are not smooth, this is due to the phase errors generated in the grating by the air-bearing stage (Aerotech). The maximum position error of the air-bearing stage is $\pm 10 \mathrm{~nm}$, while most of time the position error is a few nanometers. Fig. 5 shows a simulation result, based on coupled mode theory [11], of the reflection spectrum of a chirped grating with the same length, laser induced index change and chirp rate as those used in the experiment with result shown in Fig. 4. The position error of the stage in the simulation is random from -3 to $3 \mathrm{~nm}$.

The alignment of the laser beam focus in the fiber core is critical in order to get strong gratings with low insertion loss even with the elongation of the laser focus in the fiber core due to the 


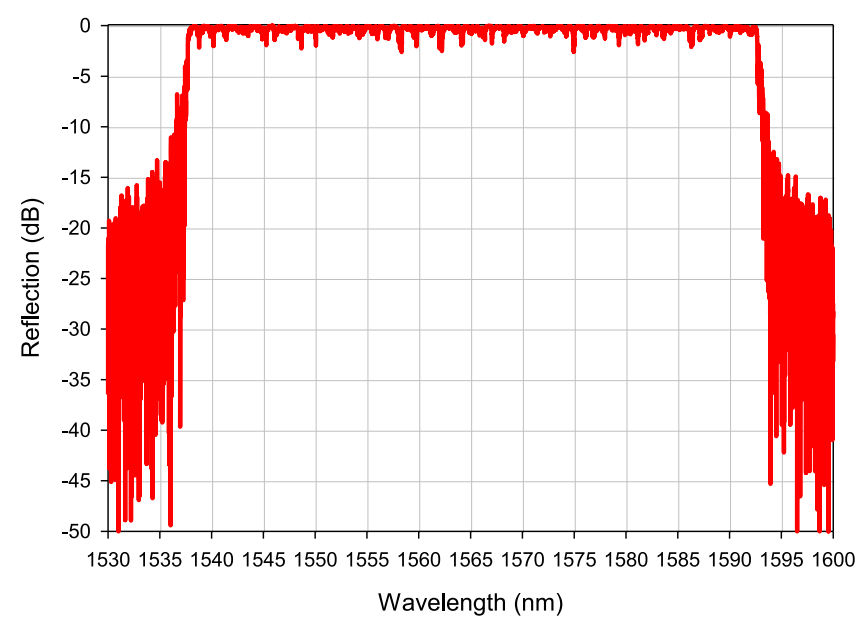

Fig. 5. Simulation of the reflection spectrum of a $260 \mathrm{~mm}$ long grating with chirp rate of $2 \mathrm{~nm} / \mathrm{cm}$. The errors of grating periods are assumed to be random from -3 to $3 \mathrm{~nm}$.

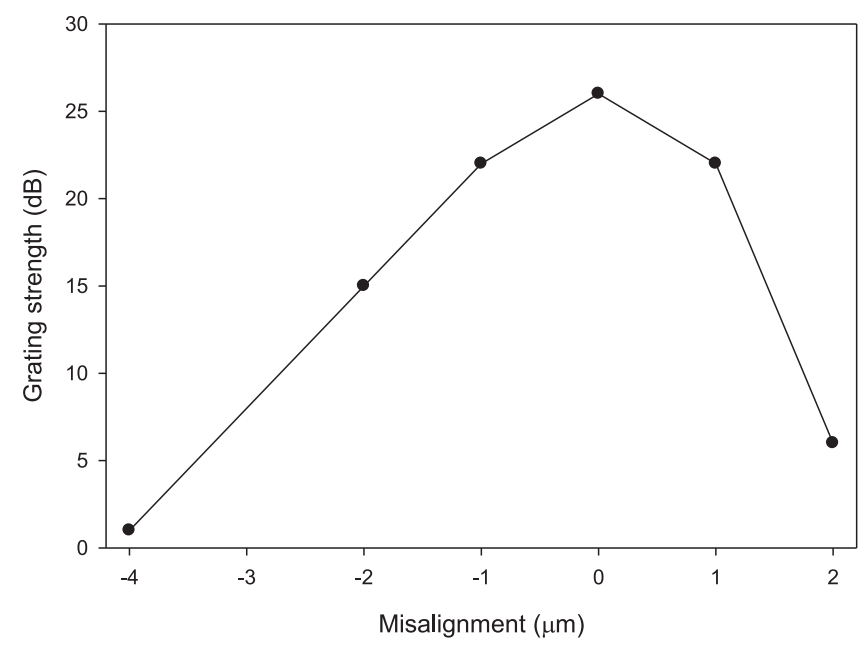

Fig. 6. Strength of $10 \mathrm{~mm}$ long gratings fabricated with the same inscription conditions except that the fiber core displaced away from the ideal alignment position.

cylindrical lens in the setup. Fig. 6 shows the changes in grating strength in transmission of $10 \mathrm{~mm}$ long gratings inscribed with the same pulse energy $(1.4 \mu \mathrm{J})$ but with the fiber shifted along the laser beam direction from the alignment position producing strongest grating. As we can see, when the fiber is shifted $2 \mu \mathrm{m}$ from the ideal position, the drop of grating strength is significant. In this work, the stages we used are ANT130-060-L (Aerotech) with a resolution of $200 \mathrm{~nm}$.

\section{B. Type II Gratings}

Type II gratings in silica fiber have the potential for sensing at high temperatures up to $1000{ }^{\circ} \mathrm{C}$. By using the proposed technique, strong type II gratings were fabricated in SMF-28 fiber with grating periods around $1.07 \mu \mathrm{m}$ (2nd order grating for Bragg wavelengths around $1550 \mathrm{~nm}$ ). The minimum pulse energy needed to produce type II gratings in SMF-28 fiber

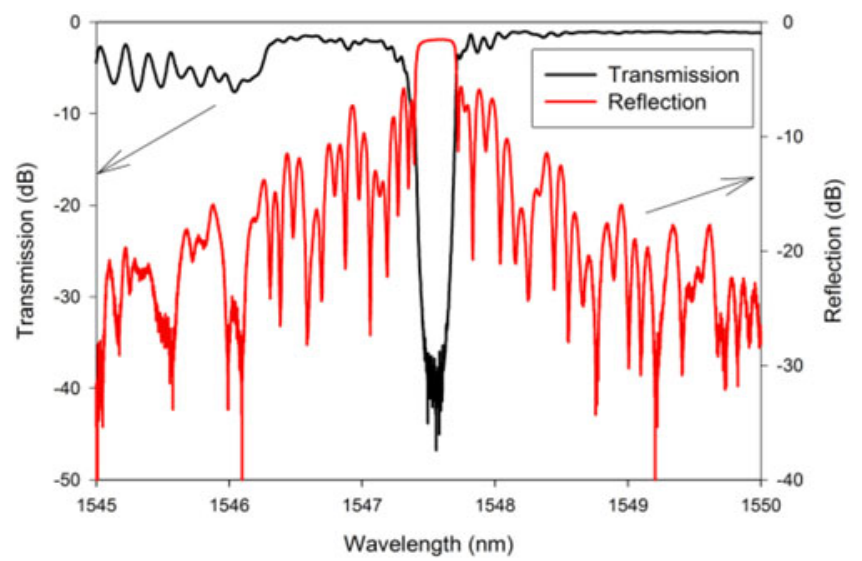

(a)

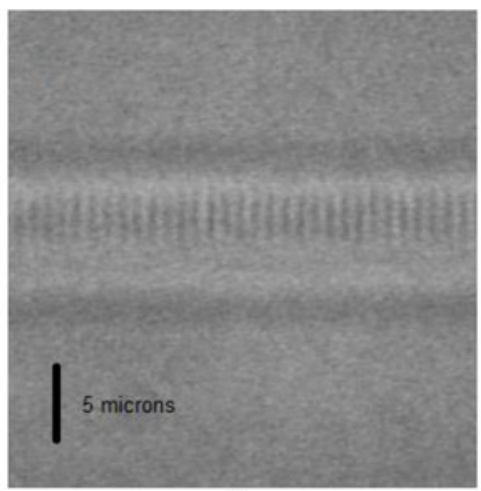

(b)

Fig. 7. Spectra and top view microscope image of a $10 \mathrm{~mm}$ long uniform type II grating in SMF-28 fiber. Small vertical lines in the middle of the core in Fig. 4(b) are perpendicular to the laser beam direction.

depends on both the objective and the cylindrical lens used in the setup. With the Nikon objective $(50 \mathrm{X} / 0.6)$, the minimum pulse energies needed to produce type II gratings are 1.3 and $1.7 \mu \mathrm{J}$ when the focal lengths of the cylindrical lenses are 1 and $0.5 \mathrm{~m}$ respectively. By using a cylindrical lens with a longer focal length $(f=1 \mathrm{~m})$, the IR laser beam was then tightly focused on the core rather than in the cladding past the core as in the type I case above. Higher insertion loss (up to $1 \mathrm{~dB} / \mathrm{cm}$ ) and strong cladding modes were produced. Fig. 7 shows the spectra and top view microscope image of a $10 \mathrm{~mm}$ long $2 \mathrm{nd}$ order grating. The laser repetition rate and pulse energy were set at $250 \mathrm{~Hz}$ and $1.4 \mu \mathrm{J}$, respectively, while the fiber moved at a speed of $267.5 \mu \mathrm{m} / \mathrm{s}$. From the top view microscope image shown in Fig. 7 it is clear that the fs-laser pulses produce lines in the fiber core with an approximate length of $5 \mu \mathrm{m}$. The side view image recorded after rotating the fiber 90 degrees along its axis is roughly the same as the top view image. The scattering loss of the grating shown in Fig. 7 is $\sim 0.8 \mathrm{~dB}$.

Due to the fact that a plane of index change can be produced by a single fs-IR pulse, the demonstrated grating fabrication setup can be easily applied to produce more exotic grating structures such as tilted gratings, which are far more complicated and time consuming to produce if the point-by-point method is used. By using a cylindrical lens with a shorter focal length $(f=0.5 \mathrm{~m})$ 


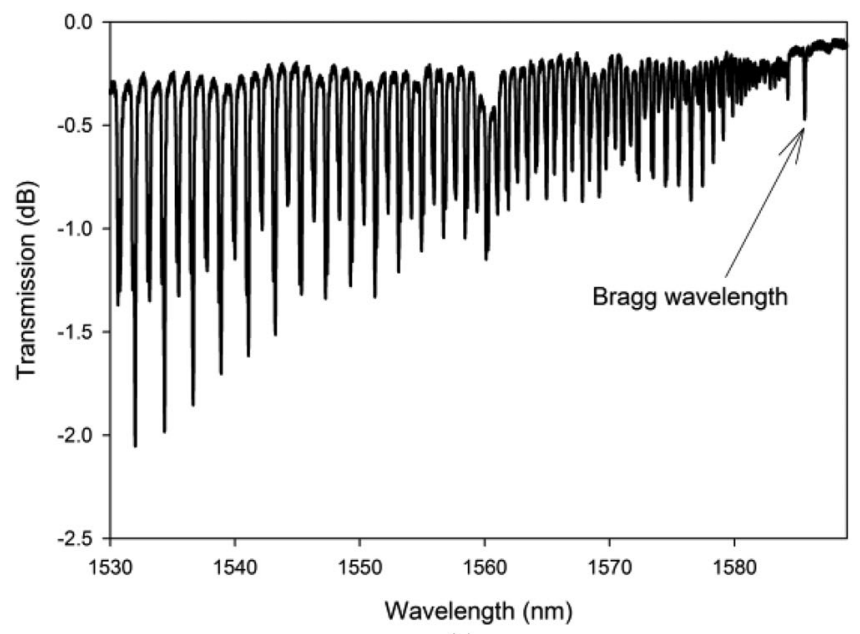

(a)

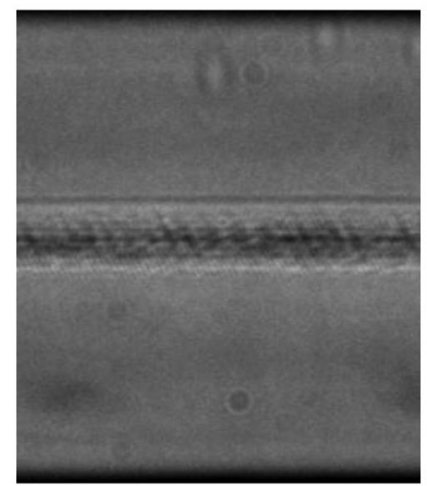

(b)

Fig. 8. Spectrum and top view microscope image of a $10 \mathrm{~mm}$ tilted grating in SMF-28 fiber.

and rotating it so that the IR laser-produced plane is not perpendicular to the fiber axis, tilted gratings with strong cladding modes can easily be made. By reducing the repetition rate to $125 \mathrm{~Hz}$ and increasing the pulse energy to $1.9 \mu \mathrm{J}$, and rotating the cylindrical lens $20^{\circ}$ from the position used to fabricate planes perpendicular to the fiber axis, a tilted grating with an interplane separation of $1.09 \mu \mathrm{m}$ is produced (see Fig. 8). The distortion of the image of the grating plane shown in Fig. 8 is due to the focusing effect of the fiber [18]. Again, a higher scattering loss $(0.5 \mathrm{~dB})$ is observed.

\section{CONCLUSION}

A simple method of fabricating both type I and type II gratings using a femtosecond IR laser was proposed and demonstrated experimentally. The proposed plane-by-plane approach was shown to be an effective way of fabricating strong, low loss and complicated type I grating structures in SMF-28 fiber without the need of $\mathrm{H}_{2}$ or $\mathrm{D}_{2}$ loading. Gratings were inscribed in free-standing fiber avoiding the use of oil-immersion objectivebased setups, which simplifies the grating inscription process and makes it easier for practical grating production.

\section{REFERENCES}

[1] S. J. Mihailov et al., "Fiber Bragg gratings made with a phase mask and 800-nm femtosecond radiation," Opt. Lett. vol. 28, no. 12, pp. 995-997, Jun. 2003.

[2] S. J. Mihailov, D. Grobnic, C. W. Smelser, P. Lu, R. W. Walker, and H. Ding, "Bragg grating inscription in various optical fibers with femtosecond infrared lasers and a phase mask," Opt. Mater. Express, vol. 1, no. 4, pp. 754-765, Aug. 2011.

[3] A. Martinez, M. Dubov, I. Khrushchev, and I. Bennion, "Direct writing of fibre bragg gratings by femtosecond laser," Electron. Lett., vol. 40, no. 19, pp. 1170-1172, Sep. 2004.

[4] G. D. Marshall, R. J. Williams, N. Jovanovic, M. J. Steel, and M. J. Withford, "Point-by-point written fiber-Bragg gratings and their application in complex grating designs," Opt. Express, vol. 18, no. 19, pp. 19844 19859, Sep. 2010.

[5] K. Zhou, M. Dubov, C. Mou, L. Zhang, V. K. Mezentsev, and I. Bennion, "Line-by-line fiber Bragg grating made by femtosecond laser," IEEE Photon. Technol. Lett., vol. 22, no. 16, pp. 1190-1192, Aug. 2010.

[6] R. J. Williams, R. G. Krämer, S. Nolte, and M. J. Withford, "Femtosecond direct-writing of low-loss fiber Bragg gratings using a continuous corescanning technique," Opt. Lett. vol. 38, no. 11, pp. 1918-1920, Jun. 2013.

[7] R. J. Williams, R. G. Krämer, S. Nolte, M. J. Withford, and M. J. Steel, "Detuning in apodized point-by-point fiber Bragg gratings: Insights into the grating morphology," Opt. Express, vol. 21, no. 22, pp. 26854-26867, Nov. 2013.

[8] R. J. Williams, N. Jovanovic, G. D. Marshall, G. N. Smith, M. J. Steel, and M. J. Withford, "Optimizing the net reflectivity of point-by-point fiber Bragg gratings: The role of scattering loss," Opt. Express, vol. 20, no. 12, pp. 13451-13456, Jun. 2012.

[9] Y. Lai, Y. Chen, and M. W.O. Cheong, "Low-loss, high reflectivity, first-order, pitch-by-pitch fiber Bragg grating fabrication in truly freestanding single-mode fiber," in Proc. J. Phys., Conf. Series 679, 2016, Art. no. 012055.

[10] A. Martinez, M. Dubov, I. Khrushchev, and I. Bennion, "Photoinduced modifications in fiber gratings inscribed directly by infrared femtosecond irradiation," IEEE Photon. Technol. Lett., vol. 18, no. 21, pp. 2266-2268, Nov. 2006

[11] T. Erdogan, "Fiber grating spectra," J. Lightw. Technol., vol. 15, no. 8, pp. 1277-1294, Aug. 1997.

[12] C. W. Smelser, S. J. Mihailov, and D. Grobnic, "Formation of Type I-IR and Type II-IR gratings with an ultrafast IR laser and a phase mask," Opt. Express, vol. 13, no. 14, pp. 5377-5386, 2005.

[13] O. Bourne, D. Rayner, P. Corkum, A. Mehendale, and A. Y. Naumov, "Method for creating optical structures in dielectrics using controlled energy deposition," U.S. Patent, US 6,884,960 B2, 2005.

[14] R. Taylor, P. Corkum, R. B. Vedula, E. Simova, D. Rayner, and C. Hnatovsky, "Fabrication of long range periodic nanostructures in transparent or semitransparent dielectrics," U.S. Patent, US 7,438,824 B2, 2008.

[15] P. Lu et al., "Plane-by-plane inscription of grating structures in optical fibers," in Proc. 25th Opt. Fiber Sensors Conf., Apr. 23, 2017, doi: 10.1117/12.2265793, Paper no. 10323-421.

[16] C. W. Smelser, S. J. Mihailov, and D. Grobnic, "Hydrogen loading for fiber grating writing with a femtosecond laser and a phase mask," Opt. Lett., vol. 29, no. 18, pp. 2127-2129, 2004.

[17] G. Rodriguez, R. L. Sandberg, Q. McCulloch, S. I. Jackson, S. W. Vincent, and E. Udd, "Chirped fiber Bragg grating detonation velocity sensing," Rev. Sci. Instrum., vol. 84, no. 1, 2013, Art. no. 015003.

[18] S. J. Mihailov et al., "UV-induced polarisation-dependent loss (PDL) in tilted fibre Bragg gratings: Application of a PDL equaliser," Inst. Elect. Eng. Proc. Optoelectron., vol. 149, no. 5/6, pp. 211-216, 2002.

Ping Lu received the B.Sc. and M.Sc. degrees from Jilin University, Changchun, China, in 1988 and 1991, respectively, and the Ph.D. degree from the University of Ottawa, Ottawa, ON, Canada, in 2002, all in physics. From 2002 to 2013, he was a Research Scientist in the Communications Research Centre Canada, Ottawa, where his research interests include the study of polarization effects in fiber-optic communication systems and the development of fiber Bragg gratings. Since 2013, he has been with National Research Council Canada as a Research Officer. His current research interests mainly include the research and fabrication of waveguide/grating structures in dielectric materials using femtosecond lasers for the applications of optical sensing and communications. 
Stephen J. Mihailov (SM'11) received the B.Sc. degree in physics from Carleton University, Ottawa, ON, Canada, in 1986, and the Ph.D. degree in physics from York University in Toronto, Toronto, ON, Canada, in 1992. From 1996 to 2013, he worked as a Research Scientist/Research Manager in the Communications Research Centre Canada, where he conducted research on high power UV-laser and fs-laser photosensitivity in optical fibers and materials as it relates to fabrication and applications of FBGs. In 2013, he joined the National Research Council Canada, where he leads the Fibre Photonics Group and its research into nonlinear applications of optical fibre, laser-material processing, and fs-laser-based manufacturing of FBGs for applications in sensing and communications. He is a co-author on more than 200 peer-reviewed international book, journal and conference publications, and is a co-inventor of 19 issued U.S. Patents. He is a Fellow of the Optical Society of America.

Huimin Ding received the Graduate Master's degree in electronic physics and devices from Southeast University, Nanjing, China, in 1990. From 2001 to 2012, she was a Research Engineer in the Communications Research Centre Canada, where she established herself as the Fibre Optic Group Expert in tapered fibre waveguides and fused biconic tapered fibre coupler components. Since 2013, she has been with the National Research Council Canada working on the development of fibre optic based chemical sensors. She is a co-inventor on 10 patents issued by the U.S. Patent office and is a co-author on 37 peer reviewed book chapter, journal, and conference publications.

Dan Grobnic received the M.Sc. degree in engineering physics and the Ph.D. degree in physics from the University Politechnica, Bucharest, Romania, in 1982 and 1996, respectively. In 2003, he became a Scientist in the Communications Research Centre Canada, where he developed FBG components for optical communications and sensing using high powered UV and femtosecond pulse duration infrared lasers. In 2013, he joined the National Research Council Canada, where he is currently working new FBG inscription techniques using fs-lasers and technology transfer of these innovations to industry. He is a coinventor of 11 issued U.S. patents and a co-author of 123 peer reviewed book chapter, journal, and conference publications.

Robert B. Walker received the B. Eng. degree in aerospace structures from Carleton University, Ottawa, ON, Canada, in 1996, and the Ph.D. degree in photonics from the Department of Physics, University of Ottawa, Ottawa, ON, Canada, in 2010, while he was working in the Communications Research Centre Canada (CRC). In 2000, he was hired by the CRC to conduct fibre optic research and develop specialized tooling for optical components used in communication networks. Since 2013, he has been with the Security and Disruptive Technologies Portfolio, National Research Council Canada. He is currently working on developing new applications of FBGs for sensors in harsh environments and technology transfer of these innovations to industry. He is a co-inventor of 10 issued U.S. patents and a co-author of 71 peer reviewed book chapter, journal, and conference publications.
David Coulas received the three year Technologist Diploma in electronics technology and chemical engineering technology from Algonquin College, Ottawa, ON, Canada, in 1983 and started work with the Communications Research Centre in 1985. In 2012, he was transferred to the National Research Council Canada as the Laboratory co-ordinator for the Fibre Photonics Facility and is responsible for maintaining all of Fibre Photonics' equipment.

Cyril Hnatovsky received the M.Sc. degree in physics from the University of Toronto, Toronto, ON, Canada, in 1999 and the Ph.D. degree in physics from the University of Ottawa, Ottawa, ON, Canada, in 2006. He is currently a Research Officer with the National Research Council Canada working on different projects related to the inscription of Bragg gratings in optical fibers. His research interests mainly include the interaction of subpicosecond laser pulses with solids including the development of advanced materials processing methodologies and their application for prototyping of photonic and microfluidic devices.

Andrei Yu Naumov received the M.Sc. degree in molecular spectroscopy from St. Petersburg State University, Saint Petersburg, Russia, in 1984, and the Ph.D. degree in solid state physics from the Ioffe Institute of the Russian Academy of Sciences, Saint Petersburg, Russia, in 1988. In 1991, he received a Humboldt Research Fellowship to study laser spectroscopy of semiconductor heterostructures at Regensburg University, Regensburg, Germany, and continued his laser/semiconductor work at Dartmouth College, Hanover, NH, USA, as a Research Associate. In 1999, he became a Principal Applications Scientist at GSI Lumonics and was responsible for the development of new processes in laser machining for PCB markets. He joined the National Research Council of Canada in 2005, managing all laser laboratories in the Extreme Photonics Group and developing new technologies for ultrashort femtosecond laser pulses and attosecond science at NRC. 